\title{
GENERACIÓN DE DATOS SINTÉTICOS CON OBJETOS DE COCINA PARA ENTRENAR REDES NEURONALES DE CONVOLUCIÓN
}

\author{
Luis Benages-Pardo ${ }^{1}$, Rubén Sagüés-Tanco ${ }^{1}$, Gonzalo López-Nicolás ${ }^{1}$ y Sergio Llorente ${ }^{2}$ \\ ${ }^{1}$ Instituto de Investigación en Ingeniería de Aragón, University of Zaragoza, Spain \\ ${ }^{2}$ BSH Home Appliances Group, Zaragoza, Spain \\ luisbenages@unizar.es, rubensagues@unizar.es,gonlopez@unizar.es, Sergio.Llorente@bshg.com
}

\begin{abstract}
Resumen
El reconocimiento de objetos ha sido un problema ampliamente analizado en el campo de la visión por computador desde hace muchos años. En este artículo utilizamos el modelo de red neuronal Mask R-CNN para detectar y segmentar algunos objetos de cocinas usando imágenes RGB. Existen algunas bases de datos públicas con los objetos segmentados y etiquetados por clases, para entrenar este tipo de redes pero normalmente no incluyen clases para aplicaciones concretas. Crear un conjunto de datos etiquetado a mano completamente es una tarea muy tediosa y larga. Proponemos un método eficiente para crear imágenes etiquetadas con poco esfuerzo combinando imágenes de fondos con objetos segmentados. Las imágenes se pueden crear manteniendo el realismo en cuanto a la posición y la escala de los objetos, o automáticamente mediante un posicionamiento aleatorio. Se presenta finalmente una comparación de los resultados obtenidos al entrenar la red con ambos conjuntos de datos creados de forma sintética.
\end{abstract}

Palabras clave: Segmentación de objetos, Mask R-CNN, Objetos de cocina, Datos sintéticos.

\section{INTRODUCCIÓN}

La detección y reconocimiento de objetos a través de redes neuronales y deep learning [1] es un tema muy estudiado en visión por computador. Conseguir este objetivo resultaría de gran utilidad en aplicaciones de automatización de equipos, robótica o aplicaciones inteligentes. Los objetos a reconocer pueden ser muy diferentes dependiendo de la aplicación deseada, desde mascotas como perros o gatos, hasta cajas de cereales o galletas entre otros. Para lograr este objetivo y reconocer objetos de distintas clases de manera robusta es necesario un sistema capaz de aprender a partir de una gran cantidad de ejemplos de objetos de cada clase en diferentes situaciones.

El reconocimiento de objetos se puede llevar a cabo usando diferentes técnicas o métodos. Sin embargo, en el aprendizaje a partir de imágenes, el uso de redes neuronales convolucionales (CNN) es la técnica más utilizada. En [2], [3] se lleva a cabo un estudio y análisis de las CNN, discutiendo sobre su eficiencia en este tipo de tareas. Otros investigadores, se centran en una evaluación exhaustiva de las CNN utilizando una arquitectura de las mismas con filtros convolucionales muy pequeños $(3 \times 3)$. Con éstos, pueden lograr una mejora significativa en la configuración [4]. Alvaro Collet et al. en [5] presentan MOPED, un algoritmo robusto y eficiente para detectar objetos en distintas posiciones sobre escenas complejas. Otros autores se centran en el reconocimiento de texto sobre imágenes. Usan redes convolucionales para localizar y analizar la posición del texto [6]. Otro método usado para la detección de objetos se muestra en [7] donde los autores describen un sistema de detección de objetos basado en la mezcla de piezas deformables de modelos multiescala. Su propuesta se apoya en nuevos métodos de entrenamiento discriminativo con datos parcialmente etiquetados. Experimentalmente, algunos autores se centran en la implementación del reconocimiento de objetos en robots. Para facilitar esta tarea, el robot es capaz de obtener un mapa 3D del entorno y localizar el nuevo objeto por comparación [8]. Kaiming $\mathrm{He}$ et al. en [9] presenta la red Mask R-CNN, sucesora de la Faster-RCNN [10]. Esta red es ampliamente utilizada para reconocimiento y localización de objetos. Su funcionamiento se basa en la generación de máscaras para segmentar los objetos entrenados en la imagen, mejorando así la localización proporcionada por los cuadros delimitadores que enmarcan dichos objetos.

Las CNN son uno de los mejores métodos existentes para el reconocimiento de objetos, pero necesitan una gran cantidad de datos etiquetados para poder aprender correctamente. Existen bases de datos públicas, como COCO [11] y PASCAL VOC2012 [12] en los que están etiquetadas una gran variedad de clases. Estos datos están etiquetados a mano y proporcionan tanto las etiquetas de clase, como las máscaras de los objetos. Uno de los inconvenientes de utilizar esta clase de datos es la poca precisión que se puede obtener si lo que se busca es detectar objetos en un entorno determinado. Otros conjuntos de datos como [13] sí que se centran en un escenario concreto, pero etiquetan los objetos únicamente con cuadros delimitadores. Para lograr una mayor precisión en un dominio específico se necesitan datos que se centren en ese tipo de escenarios concretos. De esta manera, con una tipología concreta de escenarios, y utilizando gran variedad de 
los mismos, se puede lograr un modelo robusto para una aplicación determinada, es decir, conseguir un modelo que sea capaz de generalizar detectando los objetos en cualquier escenario. Georgios Georgakis et al. en [14] proponen una alternativa al etiquetado manual. Presentan un método, trabajando con la red neuronal Faster-RCNN, donde los objetos son insertados sobre fondos aleatorios. Los objetos son seleccionados del dataset BigBird [15] y añadidos sobre las superficies de apoyo de la imagen de fondo. Debidatta et al.[16] crean datos sintéticos de una forma similar, insertando objetos de forma aleatoria sobre fondos también aleatorios.

Otros investigadores, usan modelos 3D para crear los datos con los que entrenar la red neuronal. Hao Su et al. [17] consigue datos para entrenar la red a través de modelos 3D donde puede obtener todas las vistas etiquetadas de un objeto. Otros extraen información de modelos 3D que luego pueden utilizar para reconstruir imágenes de modelos $3 \mathrm{D}$, reducir el ruido y convertir modelos 2D en modelos 3D [18]. Algunos autores utilizan modelos de objetos $3 \mathrm{D}$ como fuente de información para construir un detector de multiples clases [19]. El entrenamiento de redes neuronales a través de datos obtenidos con modelos 3D puede tener desventajas debido a la calidad de la representación de los modelos del mismo. En [20], [21] se verifica que en las CNN entrenadas con imágenes obtenidas de modelos 3D no son necesarios aspectos como textura, posición o fondo sobre el que se encuentran. Yair MovshovitzAttias et al. [22] se centran en la creación de datos semi-automáticos mediante el uso de datos sintéticos. Generan un gran conjunto de datos de coches representados con diferentes puntos de vista e investigan los efectos de la representación y el realismo de las imágenes.

Nuestro objetivo es obtener una implementación del modelo Mask R-CNN para realizar segmentación de objetos de cocina, siendo éste el primer paso en nuestra investigación para el desarrollo de un asistente de cocina capáz de guiar y reconocer el proceso de cocinado. Para ello, en este artículo se plantea un método de entrenamiento de la red con datos sintéticos con el que reducir el esfuerzo de etiquetado. Estos datos sintéticos se basan en la fusión de imágenes de objetos reales, con imágenes de fondo de cocinas también reales. Abordamos esta tarea con dos métodos diferentes: en el primero se crean imágenes posicionando distintos objetos sobre fondos de cocinas de manera realista, y el segundo método crea las imágenes realizando un pegado aleatorio de los objetos sobre el fondo. Además, hemos utilizado diferentes técnicas de procesamiento sobre la imagen, como cambios geométricos o filtros de color sobre los objetos para añadir variedad al conjunto de datos. Esto nos permite obtener escenarios en los que los objetos parecen en movimiento o hay cambios de luminosidad. En resumen, proponemos crear un conjunto de datos sintético capaz de hacer imágenes realistas de objetos en cocinas. Esto nos permite reducir el tiempo necesario para crear datos adecuados y puede ser un primer paso en la automatización del aprendizaje.

\section{BASES DEL MODELO NEURONAL}

Las redes neuronales convolucionales se pueden utilizar para un gran número de tareas tales como análisis de datos, reconocimiento o seguimiento de objetos. Nuestro proyecto se enfoca en el reconocimiento de objetos y acciones dentro del ámbito de la cocina. El objetivo principal es analizar los resultados obtenidos con datos sintéticos que presenten bajo coste de etiquetado. Nuestro trabajo se ha basado en el modelo de deep learning denominado Mask R-CNN [9] para la detección y segmentación de objetos presentes en la cocina. Esta implementación parte del framework realizado por Matterport Inc. [23] en un trabajo anterior, el cual se proporciona en código abierto bajo una licencia MIT. La arquitectura y el funcionamiento de esta red se encuentra detallado en [9] [24]. El primer bloque de la arquitectura está formado por tres sub-bloques: Backbone que extrae mapas de características de las imágenes con capas convolucionales (normalmente ResNet50 o ResNet101), Feature Pyramid Network (FPN) [25] que proporciona que las características de alto nivel estén a diferentes escalas y con información de diferentes niveles, y Region Proposal Network (RPN) introducido en [10]. Este bloque propone regiones en las que es posible encontrar un objeto de una de las clases entrenadas.

Una vez que se han obtenido las regiones propuestas, el algoritmo ROI-Align introducido en [9] se aplica para ajustar el tamaño de las regiones a la entrada del clasificador. Esto se realiza mediante un método de interpolación bilineal. Cada región propuesta se introduce en diferentes capas cabeceras de la red: un clasificador para estimar la clase a la que pertenece el objeto, una cabecera de regresión para estimar el cuadro delimitador del objeto, y otra cabecera formada por una Fully Convolutional Network [26] para estimar la máscara que segmenta el objeto. La función de coste utilizada es la suma de los errores de clase $\left(L_{c l s}\right)$, de cuadro delimitador $\left(L_{\text {bbox }}\right)$ y de máscara $\left(L_{\text {mask }}\right)$ [27].

$$
L=L_{c l s}+L_{b b o x}+L_{m a s k}
$$

\section{GENERACIÓN DEL DATOS SINTÉTICOS}

Se ha llevado a cabo una investigación sobre el estado de la materia para encontrar bases de datos ya desarrollados con el que poder entrenar el modelo neuronal descrito. Los datos de entrenamiento necesarios para 


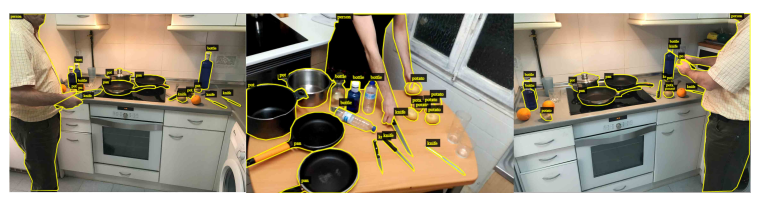

Figura 1: Ejemplo de imágenes etiquetadas con el software VIA [28]. Todas las imágenes del procedimiento manual han seguido el mismo proceso de etiquetado $\mathrm{y}$ tienen una apariencia similar.

realizar esta tarea requieren los objetos etiquetados con sus respectivas máscaras (o plantillas). De esta manera, es posible localizar automáticamente el objeto en la imagen. Un problema importante es que no existen muchas bases de datos públicas con este tipo de características. El caso de COCO [11] es uno de los más conocidos en la segmentación de objetos. Este conjunto de datos contiene más de 200.000 imágenes etiquetadas y cerca de 80 clases diferentes. Sin embargo, pocas de estas clases son de utilidad para nuestra aplicación. Por lo tanto, se ha tenido que desarrollar un conjunto de datos específico. Para crear el conjunto de datos, se han utilizado diferentes métodos con los que obtener las imágenes etiquetadas en el formato deseado. Primero se han etiquetado las imágenes a mano mediante el uso del software VGG Image Annotator tool (VIA) [28] desarrollado por Visual Geometry Group de la Universidad de Oxford. Tras comprobar que el etiquetado requerido de forma manual es una tarea muy costosa y tediosa, se optó por el desarrollo de una aplicación en Python para crear imágenes sintéticas con las que entrenar la red. Para validar los modelos entrenados con imágenes sintéticas se han utilizado las imágenes reales etiquetadas manualmente, consiguiendo así una métrica del comportamiento que tendrá el sistema en una situación real.

\subsection{DATOS ETIQUETADOS MANUALMENTE}

Para crear el conjunto de datos etiquetados a mano, se toman imágenes de cocinas reales con objetos típicos utilizados en ellas. En dichas imágenes, se han etiquetado los objetos pertenecientes a cada una de las clases que se desea entrenar. Se ha segmentado cada objeto con su máscara y se le ha asignado la etiqueta a la que pertenece. La Fig. 1 muestra unos ejemplos del proceso explicado anteriormente.

Utilizar este método para etiquetar una imagen que sea similar a las que se muestran en la Fig. 1 requiere entre 15-20 minutos de trabajo. Una vez se ha realizado el etiquetado, se empieza el entrenamiento aplicando transfer learning sobre la red Mask R-CNN de manera que se parte de los pesos pre-entrenados con los datos de COCO. De esta manera, los resultados obtenidos a pesar del pequeño número de imágenes pueden ser aceptables.

\subsection{DATOS SINTÉTICOS}

Tras los problemas comentados del etiquetado manual de imágenes reales, se desarrolló una aplicación que genera escenas sintéticas de manera similar a [16], donde las imágenes quedan etiquetadas al mismo tiempo que se crean. En este caso, la aplicación se basa en la inserción de objetos segmentados sobre fondos de cocinas como encimeras, vitrocerámicas o incluso cocinas completas. La inserción de los objetos se puede hacer aleatoriamente, obteniendo imágenes poco realistas, o colocándolos de manera realista. En ambos casos, la etiqueta de clase y la máscara del objeto son conocidas. Durante la colocación de los objetos, parámetros tales como la escala y la posición donde se encuentra el objeto se guardan para poder generar la máscara posteriormente. Estos parámetros junto a las etiquetas de la clase se almacenan en un archivo de formato JSON, consiguiendo así que las anotaciones de las imágenes ocupen poco espacio en el disco. La Fig. 2 muestra algunos ejemplos de fondos utilizados para la creación de imágenes sintéticas. Todas las imágenes, los fondos y los objetos, han sido obtenidas de imágenes de internet. Se ha intentado obtener el mayor número de ángulos de visión posible de los objetos para que el modelo sea capaz de reconocerlos independientemente de su orientación respecto de la cámara.

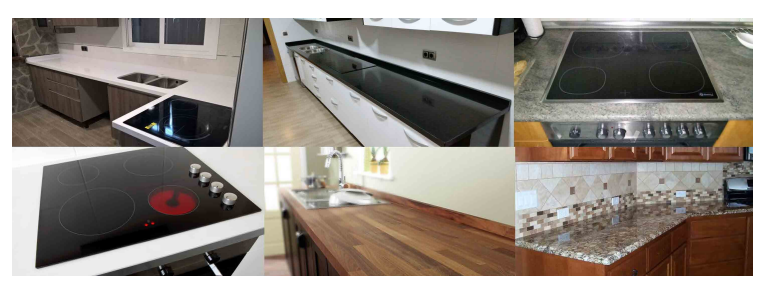

Figura 2: Ejemplo de algunos fondos utilizados para el desarrollo de los datos sintéticos. En estos fondos los objetos pueden ser posicionados de forma aleatoria o realista, según el método escogido.

En la Fig. 3 se muestran algunos de los objetos que se utilizan junto a una imagen con la máscara aplicada sobre un fondo negro. Las imágenes de los objetos se buscan sobre fondos blancos para que se puedan binarizar fácilmente mediante umbralización de color y así obtener su máscara. Algunas de las imágenes creadas con la aplicación descrita de forma realista se muestran en la Fig. 4. En estas imágenes, se mantienen escalas y orientaciones de los objetos, con respecto al fondo, para obtener escenas realistas. En la Fig. 5 se muestran algunas imágenes con los objetos posicionados de forma aleatoria, donde se observa que tienen poco parecido con una escena real. El pegado de los objetos sobre la imagen de fondo se ha realizado aplicando un fusionado entre el borde del objeto y la imagen de fondo para así intentar reducir el contraste entre las dos imágenes. Para obtener las máscaras de los objetos respecto de la imagen de fondo, se ha realizado una transformación 


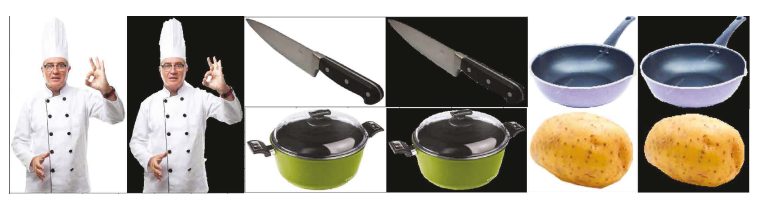

Figura 3: Ejemplo de los objetos y sus máscaras utilizados para el desarrollo de la aplicación que genera los datos sintéticos.

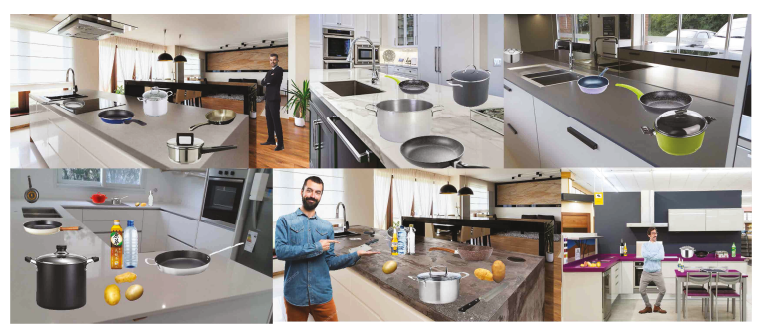

Figura 4: Ejemplos de algunas imágenes del conjunto de datos sintéticos realista creado a través de la aplicación. Los parámetros y las etiquetas de cada clase de objeto se guardan en un fichero JSON.

de las coordenadas relativas respecto de la imagen del objeto a coordenadas relativas respecto de la imágen de fondo, consiguiendo así ubicar los objetos en la nueva imagen creada. Cada vez que se añade un objeto nuevo a la imagen, se tratan las posibles oclusiones que crea el nuevo objeto sobre otros objetos anteriormente insertados, eliminando de sus máscaras guardadas los pixeles que hayan sido ocluidos.

Utilizando la aplicación descrita, el tiempo necesario para generar imágenes realistas es de unos 10-20 segundos por imagen, mientras que el tiempo para generar las imágenes con los objetos colocados aleatoriamente es de 2-3 segundos. Esto es una gran mejora con respecto al etiquetado manual que necesitaba alrededor de 15-20 minutos por imagen. Los dos tipos de datos generados de forma sintética poseen 319 imágenes. Para validar estos datos, se han usado todas las imágenes que fueron etiquetadas manualmente con la herramienta VIA tool [28] (81 imágenes). De este modo, se puede verificar el funcionamiento del modelo

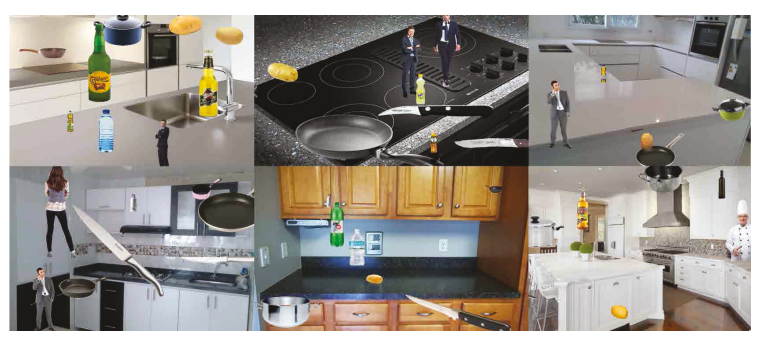

Figura 5: Ejemplos de algunas imágenes del conjunto de datos sintéticos aleatorio creado a través de la aplicación. Los parámetros y las etiquetas de cada clase de objeto se guardan en un fichero JSON. en situaciones reales. El Cuadro 1 muestra la información de los datos sintéticos de entrenamiento. En él, se muestra el número de imágenes en que aparecen los objetos de cada clase y el número total de objetos por clase. También presentamos la información de los datos etiquetados manualmente.

\section{VALIDACIÓN EXPERIMENTAL}

Una vez creados los datos sintéticos se ha comprobado su rendimiento. Para ello, se ha entrenado la red con los distintos datos independientemente. Se ha analizado la configuración genérica de la red con todos sus parámetros disponibles y se ha propuesto una configuración específica con el fin de obtener mejores resultados para nuestro problema.

\subsection{CONFIGURACIÓN EXPERIMENTAL}

El modelo presentado utiliza la red ResNet101 como Backbone para realizar la extracción de características. Como punto de partida, hemos aplicado transfer learning partiendo de los pesos pre-entrenados de $\mathrm{COCO}$ [11]. Los parámetros elegidos más relevantes han sido: learning rate de 0.001 , weight decay de 0.9 y un batch size de dos imágenes en una GPU NVIDIA Titan XP. La red ha sido entrenada en tres etapas diferentes: en la primera, se entrenan solo las cabeceras durante 60 épocas, en la segunda, se entrena desde la etapa 4 y superior en el modelo ResNet101 durante 30 épocas, y en la tercera, se entrena la red completa con un learning rate reducido un factor de 10 para el refinado de los pesos durante 15 épocas.

En el entrenamiento, se ha utilizado aumento de datos para evitar el sobreajuste de la red dando una mayor variabilidad a las imágenes. El aumento de datos se ha realizado a través de una librería de Python llamada imgaug que está disponible en [29]. Con esta librería se puede aumentar el número de imágenes para entrenar la red, alterando ligeramente las imágenes ya disponibles. La modificación de las imágenes nos ayuda también a mejorar el reconocimiento de los objetos en videos ya que se puede aplicar distintos efectos como distorsión de movimiento, o cambios de luminosidad de los objetos. Las modificaciones se han realizado sobre los objetos independientemente, y sobre el conjunto total que forma la imagen. Esto hace que el conjunto de datos tenga aún más variabilidad durante el entrenamiento de la red.

Los distintos modos de aumentos de datos aplicados a los objetos han sido: GaussianBlur, MotionBlur, LinearContrast y SigmoidContrast (Fig. 6). En el caso de las imágenes completas se han aplicado modificaciones geométricas: Affine, Fliplr, Flipud y Crop (Fig. $6)$.

Una vez la red ha sido entrenada con ambos conjuntos 
Cuadro 1: Información de los datos sintéticos

\begin{tabular}{|c|c|c|c|c|c|c|}
\hline Clase & \multicolumn{2}{|c|}{ Automático (319) } & \multicolumn{2}{|c|}{ Realista (319) } & \multicolumn{2}{|c|}{ Validación (81) } \\
\hline & Imágenes & № Objetos & Imágenes & № Objetos & Imágenes & № Objetos \\
\hline Botella & 245 & 444 & 135 & 344 & 81 & 291 \\
\hline Sartén & 233 & 390 & 212 & 346 & 78 & 143 \\
\hline Olla & 192 & 288 & 219 & 369 & 78 & 131 \\
\hline Cuchillo & 232 & 366 & 136 & 271 & 81 & 277 \\
\hline Patata & 229 & 383 & 129 & 412 & 81 & 427 \\
\hline Persona & 183 & 247 & 104 & 115 & 58 & 59 \\
\hline
\end{tabular}

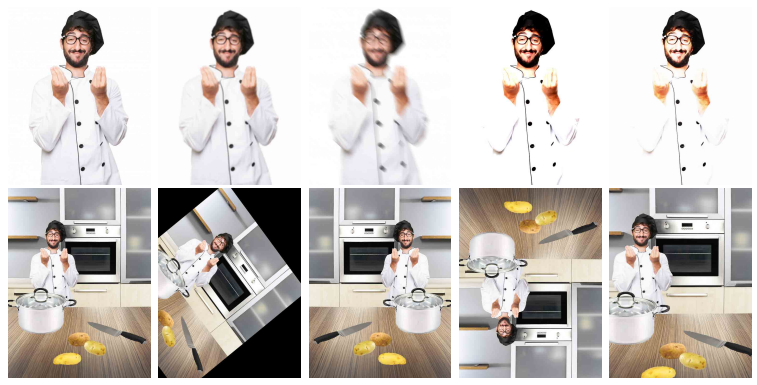

Figura 6: Ejemplo de los modos de aumento de datos que hemos aplicado sobre los objetos (arriba) y las imágenes completas (abajo) en ambos datasets.

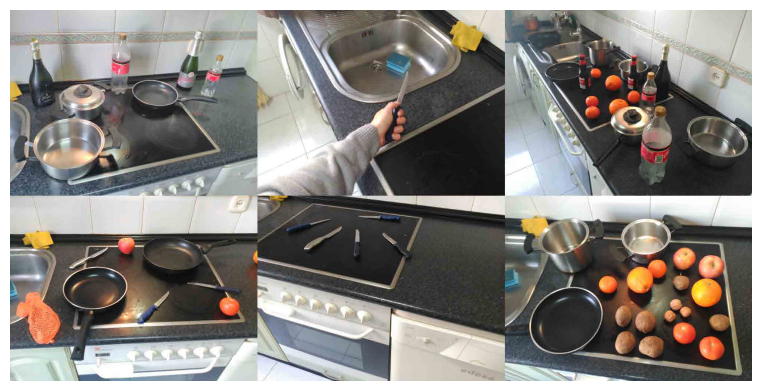

Figura 7: Ejemplo de imágenes de prueba usadas para comprobar el resultado del entrenamiento de nuestra red neuronal con ambos conjuntos de datos.

de datos sintéticos y utilizando aumento de datos sobre las imágenes, se obtiene los pesos correspondientes al conjunto de datos realista y automático. Estos pesos se comprueban sobre un conjunto de imágenes realizadas sobre cocinas reales. También, se ha utilizado un conjunto de prueba con imágenes reales que no han sido usadas ni para el entrenamiento de la red ni para su validación. Esto permite comparar visualmente la eficiencia de cada uno de los modelos entrenados con los dos tipos de datos. El conjunto de imágenes de prueba tiene las mismas clases de objetos con las que se ha entrenado la red: sartén, olla, botella, cuchillo, persona y patata. También se han introducido otros objetos como naranjas, tenedores, nueces... para comprobar los falsos positivos obtenidos. En la Fig. 7 se muestran algunos ejemplos de las imágenes usadas en este conjunto de prueba.

\section{ANÁLISIS DE LOS RESULTADOS EXPERIMENTALES}

Se presenta una comparación entre los resultados obtenidos con el modelo entrenado con imágenes realistas y el modelo entrenado con imágenes generadas automáticamente. Esta comparación se realizó calculando la métrica de precisión media (mAP), definida en PASCAL VOC 2012 [12], sobre el conjunto de validación. Esta métrica utiliza un valor umbral de Intersection Over Union (IoU) de 0.5 (50\%). Aunque habitualmente en clasificación de objetos se utiliza la matriz de confusión para analizar los resultados, en labores de segmentación el valor de mAP da mayor fiabilidad al tener en cuenta tanto la clasificación como la correcta segmentación de los objetos. Como se puede ver en el Cuadro 2, se ha calculado el AP de cada clase y la media de todas ellas es el mAP obtenido. Los resultados obtenidos con los datos realistas son mejores que los obtenidos con los datos automáticos. Esta diferencia se puede ver claramente en el caso extremo de la clase "patata". Los datos automáticos tienen un valor de 0.07 AP, mientras que el conjunto de datos realista alcanza un valor de 0.91 AP. Esto muestra que la red Mask R-CNN tiene en cuenta el realismo de las imágenes, es decir, la posición y la escala de los objetos en el entorno. Analizando los resultados obtenidos con los datos realistas se observa cómo algunas clases tienden a ser más difíciles de detectar que otras, por ejemplo la clase cuchillo. Esto puede deberse a la similitud de este objeto con otro o a la gran variedad de formas que tiene. En la Fig. 8 se pueden observar los falsos positivos y las detecciones correctas obtenidas con ambos modelos para todas las clases analizadas. Para mejorar los resultados en estas clases sería necesario introducir más imágenes con las que poder entrenar la red.

En la Fig. 9 se muestran algunos ejemplos de la segmentación obtenida sobre el conjunto de test por los dos modelos entrenados. En las imágenes se observa el resultado visual del comportamiento de los dos modelos entrenados. En estas imágenes, se puede comprobar cómo con ambos modelos se ha podido segmentar correctamente la mayoría de las clases entrenadas. Hay que tener en cuenta, que la alternativa de los datos automáticos funciona correctamente, excepto por un par de clases que producen valores bajos de mAP (Cua- 
Cuadro 2: Resultado de mAP obtenido en validación con el modelo entrenado sobre ambos datsets sintéticos

\begin{tabular}{|c|l|l|l|l|l|l|r|}
\hline Datos & \multicolumn{9}{|c|}{ AP por clase } & MAP \\
\hline & Botella & Sartén & Olla & Cuchillo & Patata & Persona & \\
Automático & 0.8 & 0.73 & $\mathbf{0 . 7 8}$ & 0.47 & 0.07 & 0.93 & 0.6306 \\
Realista & $\mathbf{0 . 8 6}$ & $\mathbf{0 . 9 1}$ & 0.68 & $\mathbf{0 . 6 8}$ & $\mathbf{0 . 9 1}$ & $\mathbf{0 . 9 6}$ & $\mathbf{0 . 8 3 4 1}$ \\
\hline
\end{tabular}
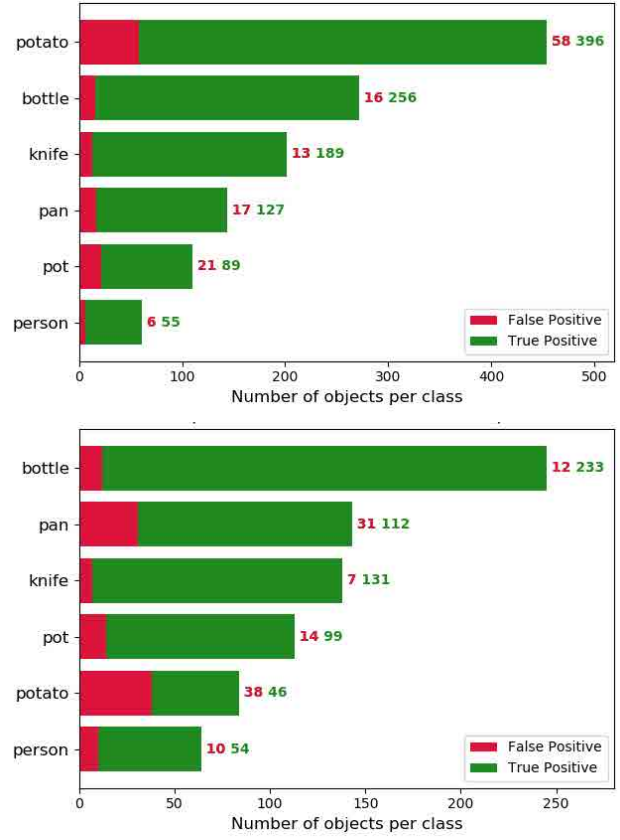

Figura 8: (Arriba) Gráfica con las detecciones correspondientes a los datos realistas, (Abajo) Gráfica con las detecciones correspondientes a los datos automáticos.

dro 2). Sin embargo, el coste de crear estas imágenes es insignificante, por lo que sería interesante en el futuro explorar si creando más imágenes con estos objetos mejora la precisión obtenida.

\section{CONCLUSIÓN}

En este artículo, se han propuesto nuevas ideas para entrenar modelos para el reconocimiento de objetos presentes en las cocinas durante el proceso de cocinado. Nuestro objetivo principal es el reconocimiento de estos objetos en imágenes y videos. Para ello, se ha adaptado la red neuronal Mask R-CNN que ha sido utilizada ampliamente en los últimos años para tareas similares. Debido a la escasa o nula disponibilidad de datos públicos con objetos de cocina que presenten las etiquetas necesarias para entrenar este tipo de red neuronal, se han desarrollado datos propios. Etiquetar imágenes a mano ha sido la primera opción, pero esto requiere invertir una gran cantidad de tiempo para el etiquetado. Por esta razón, se han generado dos conjuntos de datos sintéticos para entrenar el modelo de red, y se ha utilizado las imágenes reales etiquetadas a mano para validarlo. Para ello, se ha desarrollado una
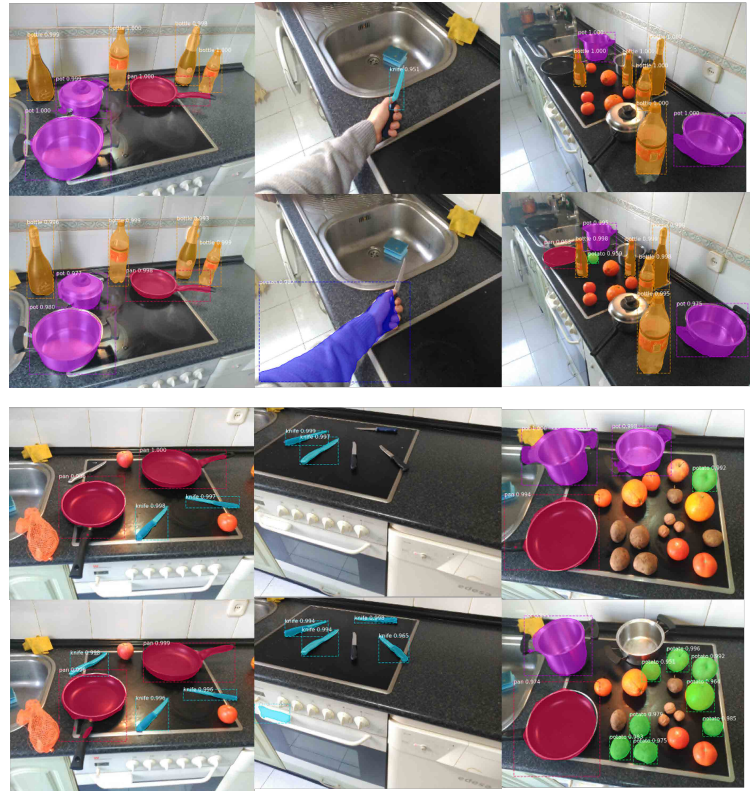

Figura 9: Ejemplos de los resultados obtenidos con el modelo de la red entrenado para ambos datasets. En la primera y tercera fila se muestran los resultados obtenidos con los datos realistas sintéticos. En la segunda y cuarta fila se muestran los resultados de datos sintéticos automáticos.

aplicación que genera imágenes realistas que reducen en gran medida el coste de etiquetado, o crea imágenes con el posicionado aleatorio de objetos de cocina sobre un fondo con menor coste de etiquetado. Se ha comparado y analizado los resultados obtenidos con ambos tipos de datos generados. Se ha podido observar que se obtienen resultados prometedores consiguiendo alcanzar buenas precisiones con una pequeña cantidad de datos. La aplicación desarrollada para crear los datos sintéticos abre una amplia gama de posibilidades en este campo, permitiendo introducir nuevas clases de objetos en la base de datos con poco esfuerzo de etiquetado, o mejorar los resultados generando mayor número de imágenes.

Como trabajo futuro, se considera la posibilidad de crear imágenes para entrenar la red mediante el renderizado de modelos 3D. Esto permitiría automatizar y recoger una gran cantidad de vistas del mismo objeto, solventando así la gran dificultad que se ha encontrado para obtener imágenes desde diferentes vistas con las que conseguir un modelo robusto.

\section{Agradecimientos}


Este trabajo ha sido financiado por el Ministerio de Ciencia, Innovación y Universidades, Gobierno de España - Unión Europea bajo el proyecto RTC-20175965-6. La tarjeta GPU Titan Xp utilizada para esta investigación fue donada por la Corporación NVIDIA.

\section{English summary}

\section{DATASET GENERATION WITH KIT- CHEN OBJECTS WITH INSTANCE SEGMENTATION TO TRAIN CONVO- LUTIONAL NEURAL NETWORK}

\begin{abstract}
Object recognition has been a wide investigated problem in computer vision for many years. In this paper, we use the Mask R-CNN neural model proposed in the state-of-the-art to detect and segment some classes of kitchen objects using RGB images. There are very few public datasets available with mask and class labels to train this kind of network, and they usually do not include classes needed for specific applications. Creating a handcraft labelled dataset with objects segmented is a very tedious and time consuming task. Thus, we propose an efficient method to generate labelled datasets with very low effort that adequately combines background images with segmented objects. The images can be created keeping the realism in the scales and positions of the objects, or they can be created automatically with the random positioning of the objects. We compare the performance of the models trained in both kinds of synthetic images.
\end{abstract}

Keywords: Object segmentation, Mask RCNN, Kitchen objects, Synthetic dataset.

\section{Referencias}

[1] I. Goodfellow, Y. Bengio, and A. Courville, Deep Learning. The MIT Press, 2016.

[2] P. Sermanet, D. Eigen, X. Zhang, M. Mathieu, R. Fergus, and Y. Lecun, "Overfeat: Integrated recognition, localization and detection using convolutional networks," in International Conference on Learning Representations (ICLR2014), CBLS, April 2014, 2014.
[3] A. Krizhevsky, I. Sutskever, and G. E. Hinton, "Imagenet classification with deep convolutional neural networks," in Advances in Neural Information Processing Systems 25 (F. Pereira, C. J. C. Burges, L. Bottou, and K. Q. Weinberger, eds.), pp. 1097-1105, Curran Associates, Inc., 2012.

[4] K. Simonyan and A. Zisserman, "Very deep convolutional networks for large-scale image recognition," in International Conference on Learning Representations, 2015.

[5] A. Collet, M. Martinez, and S. S. Srinivasa, "The moped framework: Object recognition and pose estimation for manipulation.," I. J. Robotics Res., no. 10 , pp. $1284-1306$.

[6] A. Gupta, A. Vedaldi, and A. Zisserman, "Synthetic data for text localisation in natural images," CoRR, abs/1604.06646, 2016.

[7] P. F. Felzenszwalb, R. B. Girshick, D. McAllester, and D. Ramanan, "Object detection with discriminatively trained part-based models," IEEE Trans. Pattern Anal. Mach. Intell., vol. 32, no. 9, pp. 1627-1645, 2010.

[8] S. Song, L. Zhang, and J. Xiao, "Robot in a room: Toward perfect object recognition in closed environments," CoRR, abs/1507.02703, 2015.

[9] K. He, G. Gkioxari, P. Dollár, and R. B. Girshick, “Mask R-CNN," CoRR, abs/1703.06870, 2017.

[10] S. Ren, K. He, R. Girshick, and J. Sun, "Faster R-CNN: Towards real-time object detection with region proposal networks," in Advances in Neural Information Processing Systems 28 (C. Cortes, N. D. Lawrence, D. D. Lee, M. Sugiyama, and R. Garnett, eds.), pp. 91-99, Curran Associates, Inc., 2015.

[11] T. Lin, M. Maire, S. J. Belongie, L. D. Bourdev, R. B. Girshick, J. Hays, P. Perona, D. Ramanan, P. Dollár, and C. L. Zitnick, "Microsoft COCO: common objects in context," CoRR, $a b s / 1405.0312,2014$.

[12] M. Everingham, L. Van Gool, C. K. I. Williams, J. Winn, and A. Zisserman, "The pascal visual object classes (voc) challenge," International Journal of Computer Vision, vol. 88, no. 2, pp. 303338, 2010.

[13] D. Damen, H. Doughty, G. M. Farinella, S. Fidler, A. Furnari, E. Kazakos, D. Moltisanti, J. Munro, T. Perrett, W. Price, and M. Wray, "Scaling egocentric vision: The epic-kitchens dataset," in $\mathrm{Eu}$ ropean Conference on Computer Vision (ECCV), 2018. 
[14] G. Georgakis, A. Mousavian, A. C. Berg, and J. Kosecka, "Synthesizing training data for object detection in indoor scenes," CoRR, $a b s / 1702.07836,2017$.

[15] A. Singh, J. Sha, K. S. Narayan, T. Achim, and P. Abbeel, "Bigbird: A large-scale 3d database of object instances,” pp. 509-516, May 2014.

[16] D. Dwibedi, I. Misra, and M. Hebert, "Cut, paste and learn: Surprisingly easy synthesis for instance detection," CoRR, abs/1708.01642, 2017.

[17] H. Su, C. R. Qi, Y. Li, and L. J. Guibas, "Render for CNN: viewpoint estimation in images using cnns trained with rendered $3 \mathrm{~d}$ model views," CoRR, abs/1505.05641, 2015.

[18] K. Rematas, T. Ritschel, M. Fritz, and T. Tuytelaars, "Image-based synthesis and re-synthesis of viewpoints guided by $3 \mathrm{~d}$ models," in Proceedings of the IEEE Computer Society Conference on Computer Vision and Pattern Recognition, June 2014.

[19] M. Stark, M. Goesele, and B. Schiele, "Back to the future: Learning shape models from $3 \mathrm{~d}$ CAD data," in Proceedings of the British Machine Vision Conference, pp. 106.1-106.11, BMVA Press, 2010.

[20] X. Peng, B. Sun, K. Ali, and K. Saenko, "Exploring invariances in deep convolutional neural networks using synthetic images," CoRR, $a b s / 1412.7122,2014$.

[21] B. Sun and K. Saenko, "From virtual to reality: Fast adaptation of virtual object detectors to real domains," in Proceedings of the British Machine Vision Conference, BMVA Press, 2014.

[22] Y. Movshovitz-Attias, T. Kanade, and Y. Sheikh, "How useful is photo-realistic rendering for visual learning?," CoRR, abs/1603.08152, 2016.

[23] W. Abdulla, "Mask R-CNN for object detection and instance segmentation on keras and tensorflow." https://github.com/matterport/Mask_RCNN, 2017. Online; accessed 9-May-2019.

[24] R. Anantharaman, M. Velazquez, and Y. Lee, "Utilizing mask R-CNN for detection and segmentation of oral diseases," 2018 IEEE International Conference on Bioinformatics and Biomedicine (BIBM), pp. 2197-2204, 2018.

[25] T.-Y. Lin, P. Dollár, R. B. Girshick, K. He, B. Hariharan, and S. J. Belongie, "Feature pyramid networks for object detection," 2017 IEEE Conference on Computer Vision and Pattern Recognition (CVPR), pp. 936-944, 2017.
[26] J. Long, E. Shelhamer, and T. Darrell, "Fully convolutional networks for semantic segmentation," in 2015 IEEE Conference on Computer Vision and Pattern Recognition (CVPR), pp. 3431-3440, June 2015.

[27] J. W. Johnson, "Adapting mask-RCNN for automatic nucleus segmentation," CoRR, abs/1805.00500, 2018.

[28] A. Dutta and A. Zisserman, "The VIA annotation software for images, audio and video," arXiv preprint arXiv:1904.10699, 2019.

[29] A. B. "imgaug." https://github.com/aleju/imgaug, 2018. Online; accessed 9-May-2019.

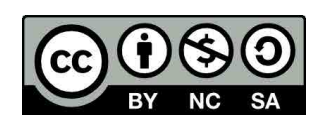

(C) 2019 by the authors. Submitted for possible open access publication under the terms and conditions of the Creative Commons Attribution CC BY-NCSA 4.0 license (https://creativecommons.org/licenses/by-ncsa/4.0/deed.es). 